OPEN ACCESS

Edited by:

Nicholas Syn,

National University of Singapore,

Singapore

Reviewed by:

Chin-Ann Johnny Ong,

National Cancer Centre Singapore,

Singapore

Jie Tian,

Shanghai JiaoTong University, China

${ }^{*}$ Correspondence:

Lucillia Bezu

Iucilliabe@gmail.com

Guido Kroemer

kroemer@orange.fr

Specialty section: This article was submitted to

Surgical Oncology,

a section of the journal

Frontiers in Oncology

Received: 24 November 2021 Accepted: 27 December 2021

Published: 14 January 2022

Citation:

Wu Chuang A, Kepp O, Kroemer G and Bezu L (2022)

Direct Cytotoxic and Indirect, Immune-Mediated Effects of Local

Anesthetics Against Cancer.

Front. Oncol. 11:821785.

doi: 10.3389/fonc.2021.821785

\section{Direct Cytotoxic and Indirect, Immune-Mediated Effects of Local Anesthetics Against Cancer}

\author{
Alejandra Wu Chuang ${ }^{1,2}$, Oliver Kepp ${ }^{1,2}$, Guido Kroemer ${ }^{1,2,3^{*}}$ and Lucillia Bezu ${ }^{1,2,4^{*}}$ \\ 1 Equipe Labellisée Par La Ligue Contre Le Cancer, Université de Paris, Sorbonne Université, INSERM UMR1138, Centre de \\ Recherche des Cordeliers, Paris, France, ${ }^{2}$ Metabolomics and Cell Biology Platforms, Gustave Roussy Cancer Campus, \\ Université Paris Saclay, Villejuif, France, ${ }^{3}$ Pôle de Biologie, Hôpital européen Georges Pompidou, AP-HP, Paris, France, \\ ${ }^{4}$ Service d'anesthésie, Gustave Roussy Cancer Campus, Villejuif, France
}

Local anesthetics are frequently employed during surgery in order to control peri- and postoperative pain. Retrospective studies have revealed an unexpected correlation between increased long-term survival and the use of local anesthetics during oncological surgery. This effect of local anesthetics might rely on direct cytotoxic effects on malignant cells or on indirect, immune-mediated effects. It is tempting to speculate, yet needs to be formally proven, that the combination of local anesthetics with oncological surgery and conventional anticancer therapy would offer an opportunity to control residual cancer cells. This review summarizes findings from fundamental research together with clinical data on the use of local anesthetics as anticancer standalone drugs or their combination with conventional treatments. We suggest that a better comprehension of the anticancer effects of local anesthetics at the preclinical and clinical levels may broadly improve the surgical treatment of cancer.

Keywords: local anesthetics, immunity, cancer, cell death, surgery

\section{INTRODUCTION}

Malignant disease remains the second cause of death worldwide. According to the World Health Organization, cancers were responsible for 10 million deaths in 2020 (1). In most cases, treatment of solid cancers relies on tumor removal by surgical excision combined with conventional therapies such as chemotherapy and radiotherapy (2). However, standard oncological surgery may promote recurrence by facilitating cancer cell dissemination due to the mechanical removal of the tumor accompanied by the stimulation of vascular endothelial growth factor (VEGF) production by the surrounding tissue (3). Moreover, surgery often induces a stress response composed of organismal metabolic changes, local inflammation and pain, thus causing an elevation of circulating

\footnotetext{
Abbreviations: ACTH, adrenocorticotropic hormone; Ca2+, calcium ion; DNMT, DNA methyltransferase; EGA, epiduralgeneral anesthesia; EGFR, epithelial growth factor receptor; GA, general anesthesia; HB-EGF, heparin-binding epidermal growth factor-like growth factor; IFN, interferon; IL, interleukin; LA, local anesthetic; MMP, matrix metalloproteinase; NK, natural killer; PCA, patient controlled analgesia; PGE2, prostaglandin E2; PVB, paravertebral block; ROS, reactive oxygen species; TGF, tumor growth factor; TNF- $\alpha$, tumor necrosis factor $\alpha$; VEGF, vascular endothelial growth factor.
} 
glucocorticoids and compromising antitumor immune responses (4-6). Finally, surgery negatively impacts on natural killer (NK) lymphocytes that spontaneously recognize and kill cancer cells and are known to play a determinant role in controlling tumor metastasis (7). Thus, we need novel adjuvant treatments during oncological surgery to optimally control pain, while limiting inflammation in order to decrease glucocorticoid stress, sustain anticancer immune responses and control residual cancer cells.

Surprisingly, several observational retrospective studies reported an improved overall survival after the use of local anesthetics (LAs) employed alone or in combination with general anesthesia during solid tumor resection. Thus, as compared to general anesthesia alone, the combination of epidural and general anesthesia, which is usually performed to relief major surgery-induced pain, was associated with a better long-term survival after abdominal and gynecological debulking (8-11). An enhancement of clinical progression-free time was also noticed after regional anesthesia after prostate, liver or breast primary tumor removal (12-14). Despite supplemental meta-analyses strengthening these positive outcomes, no guidelines emerged from these studies given their limits and weaknesses (15-18). However, rational hypotheses to explain these observations appeared in the literature, supporting the possibility of novel guidelines in oncological anesthesia.

Here we aim at discussing the main signaling pathways underlying the antitumor effect of local anesthetics. For this, we summarize published fundamental and clinical research while focusing on the mechanisms through which the immune system is activated by local anesthetics. We specifically dwell on their capacity to potentiate conventional antineoplastic therapies, hoping to improve clinical praxis in this area of oncology.

\section{LOCAL ANESTHETICS POSSESS DIRECT ANTITUMORAL ACTIVITIES}

\section{Local Anesthetics Counteract Tumor Cell Migration}

LAs such as lidocaine, ropivacaine, levobupivacaine, bupivacaine, procaine or chloroprocaine are used in clinical practice for their analgesic properties, which are explained by the blockade of voltage-gated sodium channels necessary for pain nerve conduction (19). Surprisingly, many observational preclinical studies noticed unexpected side effects of LAs on tumor cells. For instance, migration of cancer cells was profoundly impaired after LA exposure, likely due to effects on $\mathrm{Ca}^{2+}$ signaling that affect the cytoskeleton. In human triplenegative breast cancer MDA-MB-231 cells, lidocaine $(10 \mu \mathrm{M}$ or $100 \mu \mathrm{M}$ ) inhibited the CXCR4-induced $\mathrm{Ca}^{2+}$ release, leading to actin polymerization and impaired cytoskeletal remodeling (20). Lidocaine-inhibited migration and invasion are also mediated by TRPV6 downregulation that reduced $\mathrm{Ca}^{2+}$ influx in MDA-MB231 cells, prostate cancer PC-3 cells and ovarian cancer ES- 2 cells (21). Finally, infiltration of lidocaine at surgical concentrations (5-20 $\mathrm{mM}$ ) reduced cellular migration by inhibiting the shedding of heparin-binding epidermal growth factor-like growth factor from human fibrosarcoma cells and by modulating intracellular $\mathrm{Ca}^{2+}$ (22). Ropivacaine was also described to increase E-cadherin protein expression and to downregulate vimentin, which is a major intermediate filament, thus contributing to reduce metastases (23). Note that tetracaine inhibits the formation of tubulin microtentacles that are required to promote reattachment of detached breast tumor cells during metastatic dissemination (24). Taken together, these findings indicate the existence of multiple molecular mechanisms by which LAs inhibit cancer cell dissemination. It is important to point out that, despite the presence of voltage-gated sodium channels on various cancer types such as breast, colon and lung tumor cells, most of the LA-induced anti-metastatic processes may be ascribed to mechanisms that do not require the inhibition of voltage-gated sodium-channels $(22,25-27)$ Figure 1.

In addition, bupivacaine, procaine and ropivacaine are endowed with the capacity to minimize the migration of neoplastic cells by inhibiting mitochondrial function. Indeed, due to their capacity to block signaling pathways operating downstream of RhoA such as the ROCK/MLC, ERK/MAPK/ FAK and Rac1/JNK/paxillin/FAK pathways that commonly lead to apoptosis, local anesthetics inhibit the migration of cancer cells (25-28).

A non-negligible role of microRNAs in cancer regulation and cells migration was suggested in different models of solid cancers treated by LAs. Thus, ropivacaine enhances miT-520a-3p expression in gastric cancer cells, thereby inactivating WEE1 and PI3K/AKT signaling and inhibiting cell migration (29). Lidocaine showed an unexpected ability to up-regulate miR145 and miR-539 expression in gastric carcinoma MKN45 cells and in lung cancer cells, respectively. These microRNAs directly downregulate epithelial growth factor receptor (EGFR), which is a prominent target for anticancer drugs and plays a major role in tumorigenesis and cancer cell invasion $(30,31)$. In addition, procaine induces similar antiproliferative effects by up-regulating miR-133b (32).

At clinically relevant concentrations, both lidocaine and ropivacaine block cell invasion. LAs interact with the secretion of matrix metalloproteinases (MMP) such as MMP-2 and with tumor necrosis factor (TNF) $\alpha$-dependent MMP-9 involved in invasion process by inhibiting Src-dependent inflammatory signaling pathways $(33,34)$. This anti-invasive effect does not result from direct effects on the cytoskeleton but rather from the capacity of LAs to block cancer cell migration secondary to their anti-inflammatory properties. Indeed, Src protein tyrosine kinase plays a key role in the homeostasis of the endothelial barrier. Its activation by phosphorylation is induced in response to inflammation. Furthermore, surgical procedures provoke acute inflammatory process including vasodilatation, edema and loss of endothelial barrier integrity, thereby facilitating transmigration, extravasation and dissemination of tumor cells through lymphatic and vascular circulation Figure 1.

Interestingly, some LAs (lidocaine and bupivacaine) elicit an anti-invasive property at concentrations lower than those used in clinical practice $(<1 \mathrm{mM})(21,25)$. We may hypothesize that low plasma concentrations of LAs from patients receiving local or 


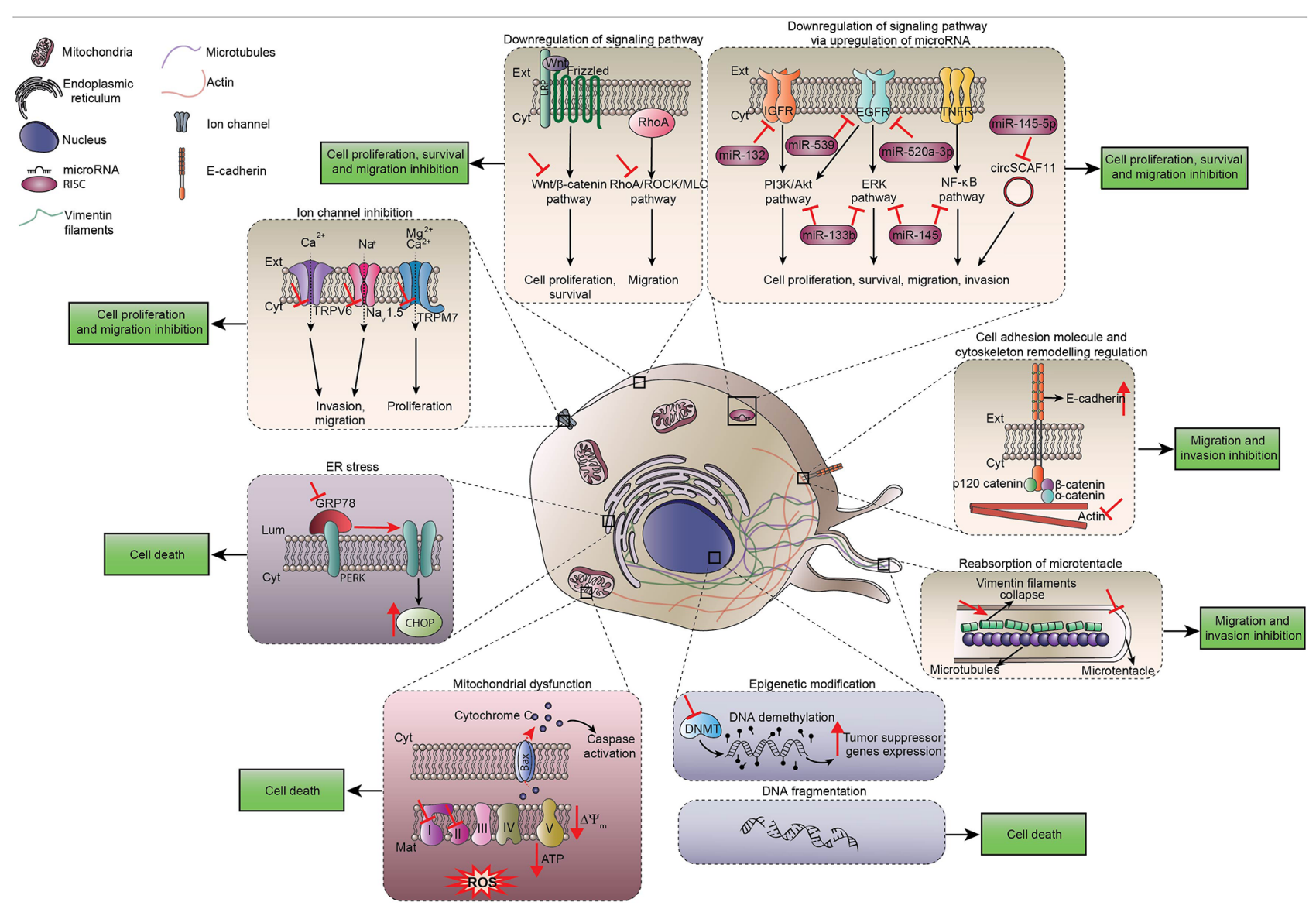

FIGURE 1 | Direct cytotoxic effects of local anesthetics. Scheme summarizing direct effects of local anesthetics on cancer cells including the regulation of signaling pathways that control proliferation, survival and migration of cancer cells. Ca2+, calcium ion; CHOP, C/EBP Homologous protein; Cyt, Cytoplasm; DNMT, DNA methyltransferase; EGFR, epidermal growth factor receptor; ER, endoplasmic reticulum; Ext, extracellular space; IGFR, insulin growth factor receptor; Mg2+, magnesium ion; Na+, sodium ion; TNFR, tumor necrosis factor receptor; ROS, reactive oxygen species; PERK, protein kinase R-like ER kinase.

regional injection of LAs could suffice to exert systemic effects on residual cancer cells, stopping their migration.

Finally, in models of tumor resection established in immunocompetent mice that have developed syngeneic transplantable EL4 lymphomas or 4T1 breast cancers, lidocaine and bupivacaine used alone or combined with general anesthesia significantly decreased spontaneous metastasis independently of the route of administration (intravenous, spinal block or local infiltration of the inoculation site) (35-38). The mechanisms accounting for these antimetastatic effects remain unclear. However, an LA-induced reduction of circulating MMP-2 levels might contribute to impair tumor cell migration (38).

\section{Local Anesthetics Inhibit Tumor Cell Proliferation}

LAs are able to stop tumor cell proliferation as indicated by the decrease in the mitotic marker Ki-67 as well as by a cell cycle arrest $(39,40)$. Most of the published data showed that this effect is concentration and time dependent (41-43). Many mechanisms may explain this process. LAs directly interfere with the advancement of the cell cycle by reducing cyclins (A2, B1, B2, D, E) and cyclin-dependent kinases expression in various models of human solid cancers (colon, lung, melanoma, thyroid, liver, breast) $(28,34,39,44-47)$. In addition, LAs induce mitochondrial dysfunction causing inhibition of respiratory chain activity and ATP production as well as a shutdown of glycolysis. This LA-induced disruption leads to mitochondrial membrane depolarization, the release of cytochrome $\mathrm{c}$ into the cytosol favoring the activation of apoptotic caspases, as well as cell damage mediated by reactive oxygen species (ROS) (48-51). Some LAs affect the DNA methylation status by modulating DNA methyltransferases (DNMT) activation in several types of cancer cell lines. The decrease in global methylation induced by LAs may restore the expression of previously silenced tumor suppressor genes and mediate growth-inhibitory effects on cancer cells $(40,52-58)$. Furthermore, some experiments suggest the implication of microRNAs in the inhibition of cancer cell proliferation $(23,29,59)$. Finally, in a model of human colorectal cancer, bupivacaine and its levorotatory enantiomer levobupivacaine promote the expression of C/EBP 
homologous protein (CHOP), which is one of the key effectors of the endoplasmic reticulum stress response (60).

\section{Local Anesthetics Promote Cancer Cell Death}

Many preclinical studies suggested the capacity of LAs to induce apoptosis after triggering the activation of tumor suppressor protein p53 (TP53) (61), DNA damage (62), dissipation of the mitochondrial transmembrane potential $(48,51,63,64)$, ROS production $(51,64,65)$ or activation of the mitogen-activated protein kinase (MAPK) pathway (64). LAs can provoke mitochondrial rupture and cause the release of pro-apoptotic molecules such as cytochrome c $(48,63,64)$ and SMAC (61). In addition, LAs upregulate the pro-apoptotic proteins Bax, Bak $(31,34,42,43,47,55,64,66)$ and down-regulate their antagonist BCL-2 $(34,42,63,64,66)$. This ultimately favors the formation of the apoptosome (composed by APAF1, caspase 9 and cytochrome c) (67) and the proteolytic activation of a range of pro-caspases $(30,34,51,61-64,68)$ including pro-caspase $3(31$, $34,42,47,48,51,63,64,66,69-71)$ and in fine the cleavage of poly (ADP-ribose) polymerase 1 , marking the apoptotic death of cancer cells $(31,51,63,64,66,67,71)$.

\section{LOCAL ANESTHETICS MAY POSSESS INDIRECT ANTITUMORAL EFFECTS BY SUSTAINING THE IMMUNE SYSTEM}

Surgery per se induces stress responses involving endocrine and metabolic reactions which generate acute inflammation and interact with the immune system (6). From incision, afferent nerve pathways stimulate catecholamine production and activate the corticotropic axis (6). The increase of plasma cortisol and catecholamine levels modifies the distribution of circulating leukocytes leading to lymphopenia and promotes the synthesis of the pro-tumoral cytokine IL-6, hence potentially enhancing tumor progression. Epinephrine and norepinephrine may act on beta-adrenergic receptors found in several tumor types such as breast, prostate or liver cancer and stimulate cancer cell proliferation and migration $(72,73)$. The adrenocorticotropic hormone (ACTH) interferes with antibody synthesis and inhibits the production of interferon (IFN) by $\mathrm{T}$ cells (74). This glucocorticoid stress is sufficient to profoundly subvert anticancer immunosurveillance in a range of murine models (4). In this context, it appears important to note that regional anesthesia by LAs injected into the epidural space provides a stable pain relief by blocking nociceptive pathways. Moreover, different neuroaxial anesthetic modalities possess the outstanding capacity to minimize glucocorticoid stress during surgery and to counteract the immunodepression induced by general anesthesia. Assessment of cortisol, epinephrine and norepinephrine in the serum and in the urine of patients after laparotomy under spinal anesthesia were significantly decreased during peri- and postoperative period compared to patients under general anesthesia (75-78). Thus, LAs could prevent the neuroendocrine stress responses resulting from oncological surgery and sustain anticancer immunity. This is strongly suggested by a preclinical study of Bar-Yosef et al., in which spinal block using bupivacaine not only controlled pain in rats during laparotomy but also attenuated the post-surgical dissemination of metastases (79) Figure 2.

Acute pain generated by surgery also compromises NK cellmediated immunity, which is in the first line of defense against tumor development $(80,81)$, and fosters $\mathrm{T}$ helper lymphocyte polarization towards a Th2 profile (82). These findings highlight the need for optimal perioperative analgesia and the necessity to strengthen the immune system. Of note, at clinically relevant concentrations lidocaine enhances the cytotoxic effect of NK cells assessed by the release of lytic granules (granzyme B and perforin) (83). In addition, the serum from patients receiving LAs during tumor resection (independently of the route of administration) was particularly competent to kill cancer cells $(84,85)$, to preserve lymphocyte proliferation and to attenuate apoptosis of peripheral blood lymphocytes. The ratio of Th1/Th2 cells inclined towards a Th1 profile with secretion of IFN- $\gamma$ (86). Finally, the level of Th17 and regulatory $\mathrm{T}$ cells (Tregs) was also significantly lower compared to the control group (87) Table 1 and Figure 2.

Another hypothesis that might explain indirect anticancer effects of LAs is their capacity to blunt surgical inflammation. Despite the employment of minimally surgical procedures, the production of pro-inflammatory cytokines (IL- $1 \beta$, IL- 6 and TNF$\alpha$ ) and the inhibition of IFN $-\gamma$ responses occur from the incision of the patient's skin (82). Inflammation is marked by major vascular and exudative phenomena (edema, diapedesis and congestion) compromising the endothelial barrier and thus facilitating the formation of new metastases. Secretion of inflammatory cytokines also stimulates MMP-9 and VEGF production in the tumor-surrounding tissue and activates Src kinase that compromises vessel barrier integrity and facilitates cancer cell migration through the extracellular matrix (94). Moreover, the cytokine IL- 6 produced in the microenvironment exerts a pro-tumor activity (95). IL-6 directly stimulates the proliferation and survival of cancer cells by stimulating the advancement of the cell cycle, the expression of anti-apoptotic molecules and angiogenesis $(72,96)$. In addition, IL-6 exerts immunosuppressive effects by inhibiting dendritic cells and lymphocytes, by activating Tregs and in fine by promoting tumor immune escape. In clinical practice, high levels of IL-6 predict chemotherapy resistance and poor prognosis in many type of cancers (97). Taken together, these data suggest that the antiinflammatory effects of LAs may contribute to sustain immune effectors and to reduce tumor progression. Indeed, several randomized controlled trials showed a significant decrease of IL1, IL-6, IL-8 and MMP-3 and-9 in the serum of patients after LA injection $(88,89,92)$. Unfortunately, the impact on clinical outcomes has not yet been investigated Table $\mathbf{1}$ and Figure 2 .

\section{LOCAL ANESTHETICS COULD IMPACT ON ONCOLOGICAL OUTCOMES}

\section{Local Anesthetics Potentiate Conventional Anticancer Treatments}

Primary tumor resection is often combined with neo-adjuvant or adjuvant anticancer treatments (chemotherapy, radiotherapy or immunotherapy) shortly before or after the surgical procedure. 


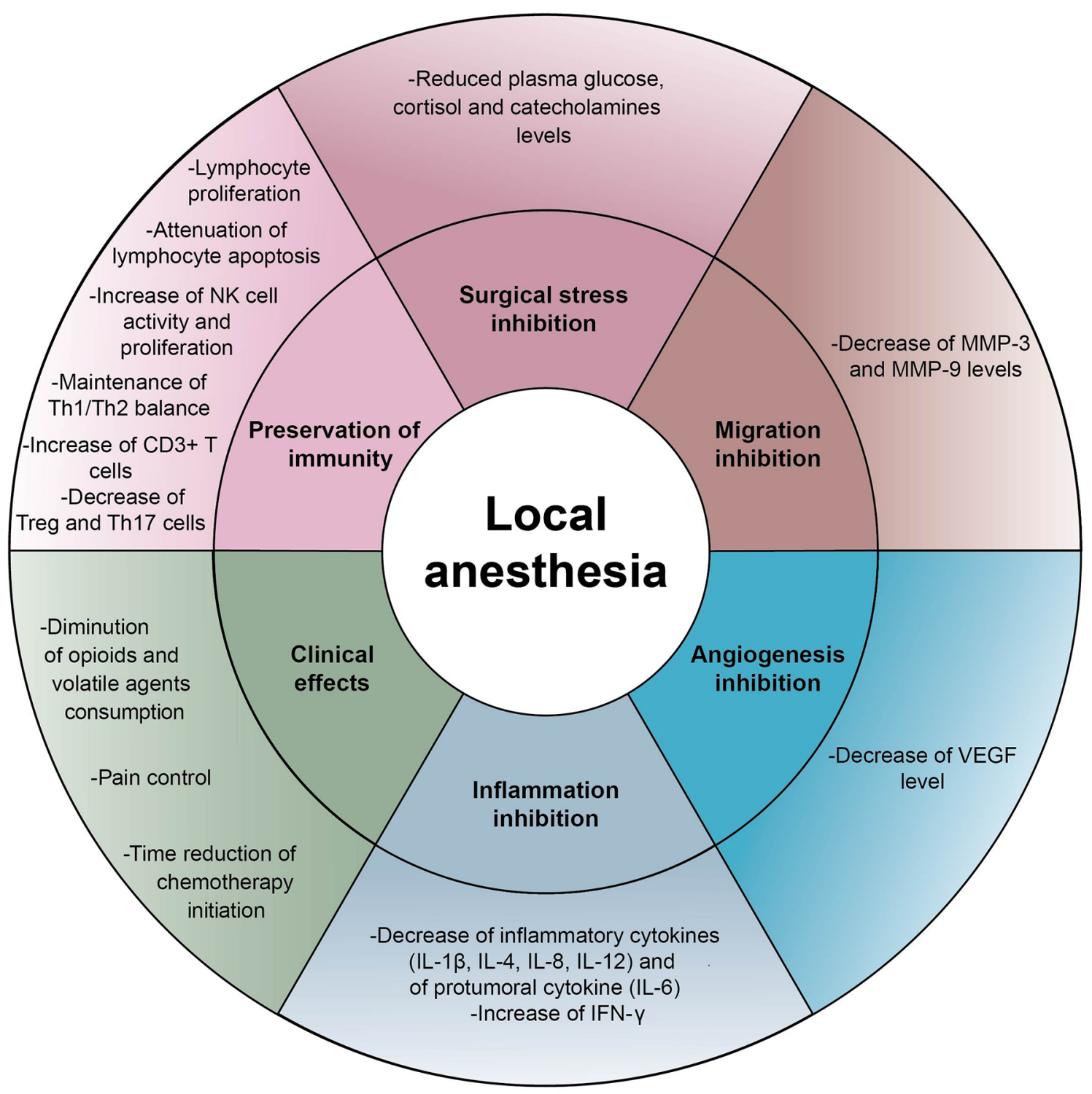

FIGURE 2 | Indirect effects of local anesthetics Schematic representation of indirect effects induced by local anesthetics on cancer cells and immune effectors: inhibition of inflammation, inhibition of cancer cell proliferation and migration, surgical stress control, reduction of neoangiogenesis, preservation of immunity and clinical effects. IFN, interferon; IL, interleukin; MMP, matrix metalloproteinase; NK, natural killer cell; VEGF, vascular endothelial growth factor.

Interestingly, LAs can sensitize cancer cells to conventional antitumor therapeutics. Thus, the cytotoxic effects of chemotherapy (with 5-fluorouracil, paclitaxel, cisplatin or carboplatin) or protein kinase inhibitors (such as vemurafenib or erlotinib) were significantly potentiated by LAs $(25,27,50,54$, 58, 68, 98, 99). Associated with 5-aza-2'-deoxycytidine, lidocaine showed additive demethylating effects in breast cancer cells (57). In vivo, the combination of cisplatin and LAs increased life span and cure rate in several mouse models $(42,100,101)$, contrasting with the observation that bosutinib reversed the anti-metastatic effect of lidocaine (38). Surprisingly, procaine demonstrated an unexpected protection against cisplatin-induced nephrotoxicity as indicated by reduced blood urea nitrogen and renal tubular degeneration (102).

\section{Local Anesthetics Improve Overall Survival After Cancer Surgery}

Many retrospective clinical studies investigated the impact of LAs on oncological prognosis. Thirteen trials suggest a potential benefit of LA injection on recurrence free survival and overall survival after cancer surgery compared to control groups. For instance, in a cohort of 588 patients undergoing primary colon cancer resection, epidural anesthesia improved the five-year survival after adjustement for relevant patient characteristics, 
TABLE 1 | Trials assessing local anesthetics on biological markers.

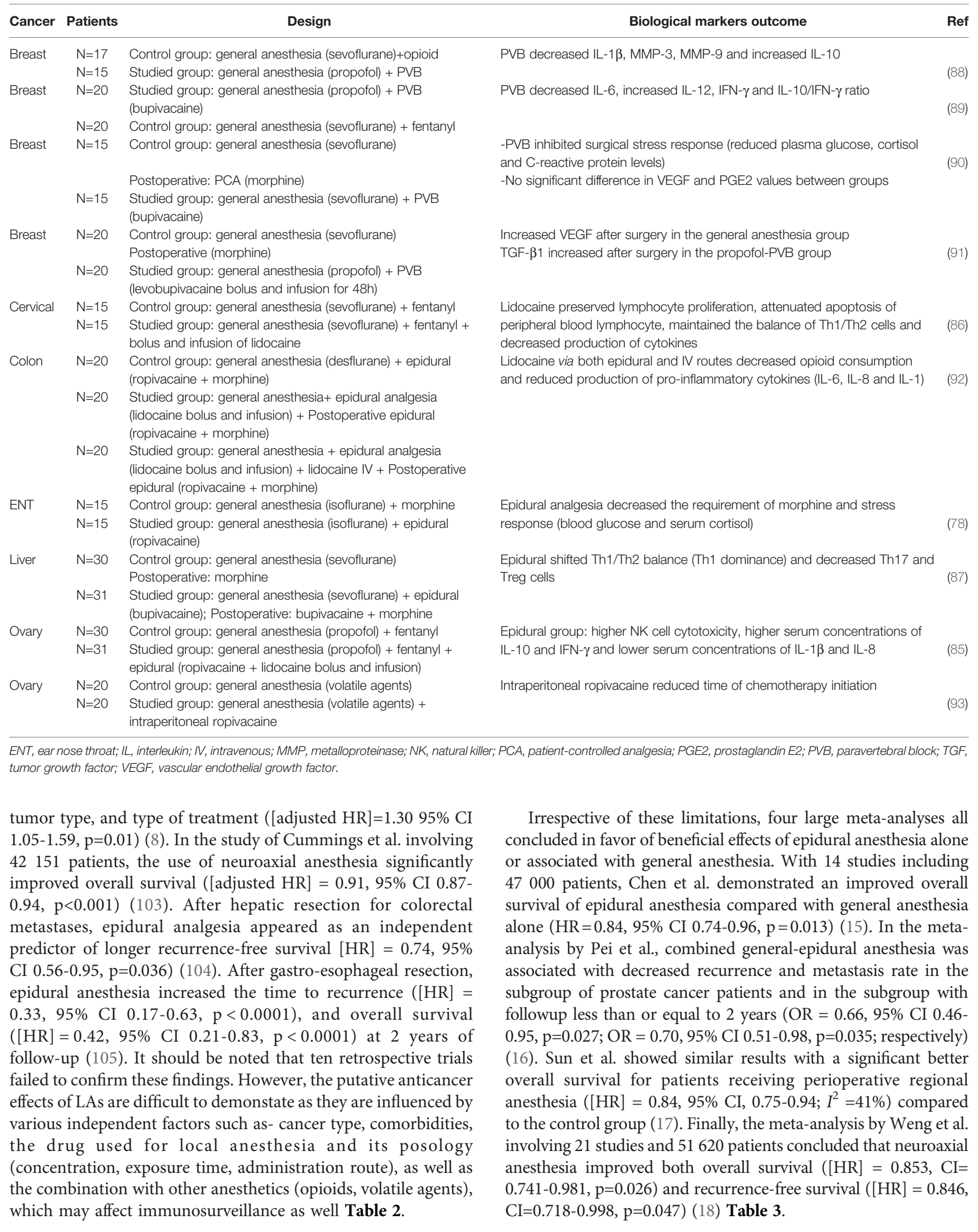


TABLE 2 | Retrospective studies assessing local anesthetics impact on cancer prognosis.

\begin{tabular}{|c|c|c|c|c|}
\hline Cancer & Patients & Design & Cancer prognosis outcome & Ref \\
\hline \multirow[t]{2}{*}{ Breast } & $\mathrm{N}=79$ & $\begin{array}{l}\text { Control group: general anesthesia (sevoflurane) } \\
\text { Postoperative: PCA (morphine) }\end{array}$ & $\begin{array}{l}\text { Studied group: lower recurrence- } \\
\text { and metastasis-free survival }\end{array}$ & (14) \\
\hline & $\mathrm{N}=50$ & $\begin{array}{l}\text { Studied group: general anesthesia (sevoflurane) + PVB (bolus and infusion of levobupivacaine for } \\
48 \text { h) }\end{array}$ & $(p=0.012)$ & \\
\hline \multirow[t]{2}{*}{ Cervical } & $\mathrm{N}=69$ & Control group: general anesthesia & Studied group: not associated & \\
\hline & $\mathrm{N}=63$ & Studied group: neuraxial anaesthesia (spinal and epidural analgesia) & $\begin{array}{l}\text { with lower cancer burden or a } \\
\text { reduced risk of tumor recurrence } \\
\text { and mortality }\end{array}$ & $(106)$ \\
\hline \multirow[t]{2}{*}{ Colon } & $N=2299$ & Control group: general anesthesia + opioid-based analgesia & No association between epidural & \\
\hline & $N=449$ & $\begin{array}{l}\text { Studied group: loading dose of lidocaine }+ \text { general anesthesia and epidural anesthesia } \\
\text { (bupivacaine with or without fentanyl for } 48-72 \mathrm{~h} \text { ) }\end{array}$ & $\begin{array}{l}\text { analgesia and recurrence or } \\
\text { death }\end{array}$ & $(107)$ \\
\hline \multirow[t]{2}{*}{ Colon } & $\mathrm{N}=668$ & Control group: general anesthesia & Peridural analgesia:not & \\
\hline & $\mathrm{N}=208$ & Studied group: epidural anesthesia & $\begin{array}{l}\text { associated with better } \\
\text { oncological outcome }\end{array}$ & (108) \\
\hline \multirow[t]{2}{*}{ Colon } & $\mathrm{N}=189$ & Control group: general anesthesia & Epidural analgesia: better 5-year & (8) \\
\hline & $\mathrm{N}=399$ & Studied group: epidural anesthesia & survival $(p=0.01)$ & \\
\hline \multirow[t]{2}{*}{ Colon } & $\mathrm{N}=253$ & Control group: general anesthesia & Epidural: lower cancer recurrence & \\
\hline & $\mathrm{N}=256$ & Studied group: epidural anesthesia & in patients older than 64 years & (109) \\
\hline \multirow[t]{2}{*}{ Colon } & $\mathrm{N}=32481$ & Control group: general anesthesia & Epidural anesthesia: improved & \\
\hline & $N=9670$ & Studied group: epidural anesthesia & survival $(p<0.001)$ & (103) \\
\hline \multirow[t]{2}{*}{ Colo-rectal } & $\mathrm{N}=93$ & $\begin{array}{l}\text { Control group: general anesthesia sevoflurane or desflurane + fentanyl and IV morphine for } 2 \text { to } 5 \\
\text { days }\end{array}$ & $\begin{array}{l}\text { Epidural anesthesia: lower } \\
\text { mortality in the sub-group of }\end{array}$ & (110) \\
\hline & $\mathrm{N}=562$ & $\begin{array}{l}\text { Studied group: general anesthesia sevoflurane or desflurane }+ \text { epidural (bolus local anesthetic and } \\
\text { fentanyl or local anesthetic alone and infusion of local anesthetic with fentanyl or local anesthetic } \\
\text { and morphine for 2-5 days) }\end{array}$ & rectal cancer $(\mathrm{p}=0.049)$ & \\
\hline \multirow[t]{3}{*}{ Colo-rectal } & $N=173$ & Control group: PCA (morphine) & No significant difference in overall & \\
\hline & $\mathrm{N}=107$ & Studied group: epidural anesthesia (Bolus and infusion of bupivacaine with fentanyl for $48 \mathrm{~h}$ ) & survival or disease-free survival at & (111) \\
\hline & $\mathrm{N}=144$ & Studied group: spinal anesthesia (bupivacaine with morphine) & 5 years & \\
\hline \multirow[t]{2}{*}{ Colo-rectal } & $\mathrm{N}=307$ & Control group: general anesthesia (isoflurane or desflurane + fentanyl) & Epidural analgesia: greater long- & (9) \\
\hline & $\mathrm{N}=442$ & Studied group: general anesthesia (isoflurane or desflurane + fentanyl) + epidural analgesia & term survival $(p<0.02)$ & \\
\hline \multirow{2}{*}{$\begin{array}{l}\text { Colo-rectal + } \\
\text { liver } \\
\text { metastases }\end{array}$} & $\mathrm{N}=120$ & Control group: IV anesthesia & Epidural anesthesia: improved & \\
\hline & $\mathrm{N}=390$ & Studied group: epidural anesthesia & $\begin{array}{l}\text { five-year recurrence free survival } \\
(p=0.036)\end{array}$ & (104) \\
\hline \multirow{2}{*}{$\begin{array}{l}\text { Gastro- } \\
\text { oeso- } \\
\text { phageal }\end{array}$} & $N=140$ & Control group: general anesthesia (sevoflurane or propofol infusion) + IV opioid analgesia & Epidural was associated with & \\
\hline & (total) & $\begin{array}{l}\text { Studied group: general anesthesia (sevoflurane or propofol) + epidural anesthesia (bupivacaine } \\
\text { bolus + infusion with morphine for } 96 \mathrm{~h} \text { ) }\end{array}$ & $\begin{array}{l}2 \text {-year recurrence and overall } \\
\text { survival benefit }(p<0.0001)\end{array}$ & (105) \\
\hline \multirow[t]{2}{*}{ ENT } & $N=160$ & Control group: general anesthesia + morphine & Epidural anesthesia:increased & \\
\hline & $N=111$ & Studied group: general anesthesia + epidural anesthesia & $\begin{array}{l}\text { cancer-free survival }(p=0.04) \text { and } \\
\text { overall survival }(p=0.03)\end{array}$ & (112) \\
\hline \multirow[t]{2}{*}{ Liver } & $\mathrm{N}=244$ & $\begin{array}{l}\text { Control group: general anesthesia (sevoflurane or propofol) + sufentanil + nonsteroidal anti- } \\
\text { inflammatory drugs }\end{array}$ & $\begin{array}{l}\text { Local anesthetic increased } \\
\text { recurrence free survival }(p=0.002)\end{array}$ & (12) \\
\hline & $\mathrm{N}=245$ & Studied group: lidocaine+nonsteroidal anti-inflammatory drugs & and overall survival $(\mathrm{p}=0.036)$ & \\
\hline Melanoma & $\begin{array}{l}\mathrm{N}=221 \\
\mathrm{~N}=52\end{array}$ & $\begin{array}{l}\text { Control group: general anesthesia (isoflurane or propofol) + sufentanil or remifentanil } \\
\text { Studied group: spinal anesthesia (bupivacaine) }\end{array}$ & $\begin{array}{l}\text { Spinal anesthesia: a trend of } \\
\text { better cumulative survival rate }\end{array}$ & (113) \\
\hline \multirow[t]{5}{*}{ NSCLC } & NA & $\begin{array}{l}\text { Control group: general anestheisa (isoflurane, sevoflurane or desflurane) + IV opioid analgesia; } \\
\text { postoperative PCA (hydromorphone, fentanyl or morphine) }\end{array}$ & $\begin{array}{l}\text { No difference on recurrence-free } \\
\text { survival or overall survival }\end{array}$ & (114) \\
\hline & & Studied group: general anesthesia (isoflurane, sevoflurane or desflurane) + IV opioid analgesia & & \\
\hline & & $\begin{array}{l}\text { Postoperative: epidural (bupivacaine }+ \text { fentanyl or bupivacaine }+ \text { hydromorphone or ropivacaine } \\
\text { and fentanyl) }\end{array}$ & & \\
\hline & & Studied group: general anesthesia (isoflurane, sevoflurane, or desflurane) + IV opioid analgesia & & \\
\hline & & $\begin{array}{l}\text { Postoperative: epidural/PCA: bupivacaine + fentanyl or bupivacaine + hydromorphone or } \\
\text { ropivacaine + fentanyl }\end{array}$ & & \\
\hline \multirow[t]{2}{*}{ Ovary } & $\mathrm{N}=37$ & Control group: general anesthesia (sevoflurane or isoflurane) + PCA fentanyl & Epidural anesthesia: greater 3- & (10) \\
\hline & $\mathrm{N}=106$ & Studied group: epidural anesthesia (Infusion of bupivacaine or ropivacaine and morphine for $48 \mathrm{~h}$ ) & $\begin{array}{l}\text { and } 5 \text {-year overall survival rates } \\
(\mathrm{p}=0.043)\end{array}$ & \\
\hline \multirow[t]{3}{*}{ Ovary } & $\mathrm{N}=43$ & Control group: general anesthesia (volatile + fentanyl) & Epidural anesthesia: not & \\
\hline & & Postoperative: ketorolac and PCA (morphine) & associated with improved overall & (115) \\
\hline & $\mathrm{N}=37$ & $\begin{array}{l}\text { Studied group: general anesthesia +epidural anesthesia (bolus of bupivacaine with or without } \\
\text { fentanyl); Postoperative: ketorolac and epidural for } 48 \mathrm{~h}\end{array}$ & survival or time to recurrence & \\
\hline Pancreas & $\begin{array}{l}\mathrm{N}=2239 \\
\text { (total) }\end{array}$ & $\begin{array}{l}\text { Control group: general anesthesia (sevoflurane) + epidural analgesia (ropivacaine) } \\
\text { Studied group:lidocaine bolus + continuous infusion + general anesthesia (sevoflurane) + epidural } \\
\text { analgesia (ropivacaine); }\end{array}$ & $\begin{array}{l}\text { Lidocaine group:longer overall } \\
\text { survival }(p=0.013)\end{array}$ & (11) \\
\hline
\end{tabular}


TABLE 2 | Continued

\begin{tabular}{|c|c|c|c|c|}
\hline Cancer & Patients & Design & Cancer prognosis outcome & Ref \\
\hline Prostate & $\begin{array}{l}N=123 \\
N=102\end{array}$ & $\begin{array}{l}\text { Control group: general anesthesia(propofol) + fentanyl } \\
\text { Postoperative: PCA (morphine) } \\
\text { Studied group: general anesthesia (propofol) }+ \text { fentanyl } \\
\text { Postoperative: local anesthetic infusion for } 48-72 \mathrm{~h}\end{array}$ & $\begin{array}{l}\text { Epidural anesthesia: lower risk of } \\
\text { recurrence }(p=0.012)\end{array}$ & (13) \\
\hline Prostate & $\begin{array}{l}N=158 \\
N=103\end{array}$ & $\begin{array}{l}\text { Control group: general anesthesia (isoflurane) + fentanyl; Postoperative: ketorolac + paracetamol } \\
\text { Studied group: general anesthesia (isoflurane) + Epidural (bupivacaine) + fentanyl }\end{array}$ & $\begin{array}{l}\text { Epidural analgesia: improved } \\
\text { clinical progression-free survival } \\
(p=0.002)\end{array}$ & (116) \\
\hline Prostate & $\begin{array}{l}N=533 \\
N=578\end{array}$ & $\begin{array}{l}\text { Control group: intravenous analgesia } \\
\text { Studied group: epidural analgesia }\end{array}$ & $\begin{array}{l}\text { Epidural analgesia:not associated } \\
\text { with a significant effect }\end{array}$ & (117) \\
\hline Visceral & $\begin{array}{l}N=63 \\
N=69\end{array}$ & $\begin{array}{l}\text { Control group: general anesthesia (isoflurane + fentanyl); } \\
\text { Postoperative: morphine } \\
\text { Epidural group: bupivacaine + general anesthesia (isoflurane); postoperative: bupivacaine + } \\
\text { morphine }\end{array}$ & $\begin{array}{l}\text { A trend in favor of epidural } \\
\text { anesthesia was observed for } \\
\text { recurrence free survival }\end{array}$ & $(118)$ \\
\hline
\end{tabular}

$I V$, intravenous; PCA, patient-controlled analgesia; PVB, paravertebral block.

$P C A$, patient-controlled analgesia; $N$, intravenous.

TABLE 3 | Meta-analyses assessing local anesthetics impact on cancer prognosis.

\begin{tabular}{|c|c|c|c|c|}
\hline Cancer & Patients & Design & Cancer prognosis outcome & Ref \\
\hline Solid & 14 studies & Control group: general anesthesia & Epidural anesthesia improved overall survival ( $p=0.013)$. & \\
\hline tumors & $\begin{array}{l}(47000 \\
\text { patients) }\end{array}$ & $\begin{array}{l}\text { Studied group: epidural anesthesia } \\
\text { with or without general anesthesia }\end{array}$ & & (15) \\
\hline $\begin{array}{l}\text { Solid } \\
\text { tumors }\end{array}$ & $\begin{array}{l}10 \text { studies } \\
(3254 \\
\text { patients) }\end{array}$ & $\begin{array}{l}\text { Control group: general anesthesia } \\
\text { Studied group: combined general- } \\
\text { epidural anesthesia }\end{array}$ & $\begin{array}{l}\text { Combined general-epidural anesthesia was associated with decreased recurrence }(p=0.027) \text { and } \\
\text { metastasis rate }(p=0.035) \text { within the subgroup of prostate cancer patients and the subgroup with } \\
\text { follow-up less than or equal to } 2 \text { years }\end{array}$ & $(16)$ \\
\hline $\begin{array}{l}\text { Solid } \\
\text { tumors }\end{array}$ & $\begin{array}{l}20 \text { studies } \\
\text { (NA) }\end{array}$ & $\begin{array}{l}\text { Control group: general anesthesia } \\
\text { Studied group: perioperative } \\
\text { regional anesthesia }\end{array}$ & $\begin{array}{l}\text { Perioperative regional anesthesia associated with improved overall survival }([\mathrm{HR}]=0.84,95 \% \mathrm{Cl} \text {, } \\
\left.0.75-0.94 ; /^{2}=41 \%\right)\end{array}$ & $(17)$ \\
\hline $\begin{array}{l}\text { Solid } \\
\text { tumors }\end{array}$ & $\begin{array}{l}21 \text { studies } \\
(51620 \\
\text { patients) }\end{array}$ & $\begin{array}{l}\text { Control group: general anesthesia } \\
\text { Studied group: neuroaxial } \\
\text { anesthesia combined with or } \\
\text { without general anesthesia }\end{array}$ & Neuroaxial anesthesia improved overall survival $(p=0.026)$ and recurrence-free survival $(p=0.047)$ & $(18)$ \\
\hline
\end{tabular}

Finally, among 11 prospective randomized controlled trials, two studies reported a better disease-free survival after epidural anesthesia (ropivacaine or bupivacaine) associated with intravenous or volatile agents during colon $(\mathrm{p}=0.012)$ or bladder tumor resection $(\mathrm{p}=0.02)$ compared to general anesthesia alone $(119,120)$. One study investigated the antitumor activity of patient sera after levobupivacaine infiltration during breast cancer resection. A significant blockade of MDA-MB-231 breast carcinoma cells was observed $(\mathrm{p}=0.01)$ (121). A better survival after hepatectomy was also noticed after infiltration of ropivacaine close to the incision site $(\mathrm{p}=0.029)$ (122). However, other trials failed to confirm these findings, perhaps due to a lack of power and major confusion bias compromising data analyses (injection of multiple different anesthetic agents, inclusion of cancers at different stages, loss of patients due to deficient followup, heterogenous groups...). Table 4 Multicenter randomized controlled trials with high quality of methodology are urgently awaited to definitevely conclude on the potential benefit of LAs on oncological outcomes.

Until now, no guidelines and no recommendations in oncoanesthesia are available to guide clinical practice. Indeed, most of the results issued from clinical studies are not convincing enough to elaborate new guidelines due to a lack of power, presence of bias, heterogeneity of groups and the combined use of various anesthetics that exert conflicting effects on tumor cells. However, based on the sheer number of prospective multicenter randomized controlled trials, we may expect the translation of preclinical data into the clinics for the near future. Thus, we anticipate that Phase III clinical trials will confirm that, beyond their useful analgesic properties, local anesthetics exert antitumor effects, meaning that their use will be approved for this additional indication.

\section{DISCUSSION}

Oncological surgery generates neuroendocrine stress, inflammation and acute pain responsible for immunosuppression, hence impacting on the antitumor immune response $(4,83)$. The manipulation of the tumor by the surgeon, vascular invasion and the peri-operative synthesis of VEGF also promote the migration and proliferation of residual cancer cells and thus, future metastatic recurrence (131).

The impact of local anesthetics on cancer and its recurrence after surgery has spurred a wave of interest over the last decade. Two recent reviews covering this field have been published $(132,133)$. 
TABLE 4 | Randomized controlled trials assessing local anesthetics impact on cancer prognosis.

\begin{tabular}{|c|c|c|c|c|}
\hline Cancer & Patients & Design & Cancer prognosis outcome & Ref \\
\hline \multirow[t]{3}{*}{ Bladder } & $N=150$ & Control group: general anesthesia (sevoflurane)+fentanyl & Local anesthesia: longer disease-free survival $(p=0.02)$ & \\
\hline & & Postoperative (morphine) & & $(119)$ \\
\hline & $N=510$ & Studied group (propofol) +lidocaine+ epidural (ropivacaine) & & \\
\hline \multirow[t]{2}{*}{ Breast } & $\mathrm{N}=11$ & $\begin{array}{l}\text { Control group: general anesthesia (sevoflurane) + morphine postoperative: PCA } \\
\text { (morphine) }\end{array}$ & $\begin{array}{l}\text { Patient serum from studied group reduced MDA-MB- } \\
231 \text { breast carcinoma cell proliferation }(p=0.01)\end{array}$ & $(121)$ \\
\hline & $\mathrm{N}=11$ & $\begin{array}{l}\text { Studied group: general anesthesia (propofol) + PVB (bolus and infusion of } \\
\text { levobupivacaine) }\end{array}$ & & \\
\hline \multirow[t]{2}{*}{ Breast } & $\mathrm{N}=30$ & Control group: general anesthesia (volatile anesthetic) & No difference between groups & \\
\hline & $\mathrm{N}=30$ & $\begin{array}{l}\text { Studied group: general anesthesia (volatile anesthetic) + PVB (ropivacaine bolus and } \\
\text { infusion) }\end{array}$ & & $(123)$ \\
\hline \multirow[t]{2}{*}{ Breast } & $N=1065$ & Control group: general anesthesia (sevoflurane) & No difference between groups & \\
\hline & $N=1043$ & Studied group: general anesthesia (propofol) + PVB & & $(124)$ \\
\hline \multirow[t]{3}{*}{ Breast } & $\mathrm{N}=58$ & Control group: general anesthesia (propofol) & No difference between groups & \\
\hline & $\mathrm{N}=56$ & Studied group: general anesthesia + single injection PVB (ropivacaine) & & $(125)$ \\
\hline & $\mathrm{N}=59$ & Studied group: general anesthesia + continuous-PVB (ropivacaine for $72 \mathrm{~h}$ ) & & \\
\hline \multirow[t]{2}{*}{ Colon } & $\mathrm{N}=92$ & Control group: general anesthesia (isoflurane)+ fentanyl & Epidural improved survival in patients without & \\
\hline & $\mathrm{N}=85$ & $\begin{array}{l}\text { Studied group: general anesthesia (isoflurane) + fentanyl + epidural group } \\
\text { (bupivacaine) }\end{array}$ & metastases $(p=0.012)$ & $(120)$ \\
\hline \multirow[t]{2}{*}{$\begin{array}{l}\text { Colon } \\
\text { Rectum }\end{array}$} & $\mathrm{N}=30$ & $\begin{array}{l}\text { Control group: general anesthesia (propofol+ remifentanyl); postoperative: PCA } \\
\text { fentanyl }\end{array}$ & $\begin{array}{l}\text { No difference for postoperative NK cell cytotoxicity } \\
\text { and IL-2, recurrence or metastasis }\end{array}$ & $(126)$ \\
\hline & $\mathrm{N}=30$ & $\begin{array}{l}\text { Studied group: general anesthesia (propofol and remifentanyl) + surgical wound } \\
\text { infiltration of ropivacaine }\end{array}$ & & \\
\hline \multirow[t]{3}{*}{ Liver } & $\mathrm{N}=20$ & Control group: tramadol injections & Ropivacaine increased postoperative survival & \\
\hline & $\mathrm{N}=20$ & Studied group: local incision analgesia (ropivacaine bolus + infiltration) & $(p=0.029)$ & $(122)$ \\
\hline & $N=20$ & Studied group: PCA (fentanyl) & & \\
\hline \multirow[t]{2}{*}{ Lung } & $N=200$ & $\begin{array}{l}\text { Control group: general anesthesia (propofol/sevoflurane+ sufentanyl/remifentanyl); } \\
\text { postoperative: PCA morphine }\end{array}$ & $\begin{array}{l}\text { No difference between groups for recurrence-free and } \\
\text { overall survival }\end{array}$ & $(127)$ \\
\hline & $N=200$ & $\begin{array}{l}\text { Studied group: general anesthesia (propofol/sevoflurane+ sufentanyl/remifentanyl)+ } \\
\text { epidural anesthesia (ropivacaine) }\end{array}$ & & \\
\hline \multirow[t]{2}{*}{ Prostate } & $\mathrm{N}=50$ & Control group: general anesthesia; postoperative: morphine & No difference between groups & \\
\hline & $\mathrm{N}=49$ & Studied group: general anesthesia + ropivacaine bolus and infusion with fentanyl & & $(128)$ \\
\hline \multirow{2}{*}{$\begin{array}{l}\text { Solid } \\
\text { tumors }\end{array}$} & $\mathrm{N}=216$ & Control group: general anesthesia; postoperative: opioid-based analgesia & No difference between groups & \\
\hline & $N=230$ & $\begin{array}{l}\text { Studied group: general anesthesia + epidural group (bupivacaine or ropivacaine); } \\
\text { postoperative: continous bupivacaine or ropivacaine + fentanyl or pethidine }\end{array}$ & & $(129)$ \\
\hline \multirow[t]{2}{*}{$\begin{array}{l}\text { Solid } \\
\text { tumors }\end{array}$} & $\mathrm{N}=822$ & $\begin{array}{l}\text { Control group: general anesthesia (propofol/sevoflurane+ sufentanyl/remifentanyl/ } \\
\text { fentanyl); postoperative: PCA morphine }\end{array}$ & No difference between groups for overall survival & $(130)$ \\
\hline & $\mathrm{N}=772$ & $\begin{array}{l}\text { Studied group: general anesthesia (propofol/sevoflurane+ sufentanyl/remifentanyl/ } \\
\text { fentanyl)+ epidural anesthesia (ropivacaine) }\end{array}$ & & \\
\hline
\end{tabular}

PCA, patient-controlled analgesia; NK, natural killer; PVB, paravertebral block.

In the present article we attempted to synthesize the current preclinical and clinical state of the art, while evoking the capacity of local anesthetics to stimulate anticancer immune responses, thereby potentiating the efficacy conventional anticancer therapies. Particular emphasis has been laid on the difference direct effects impacting on cancer cells and indirect, immunemediated effects controlling residual tumor cells that mediate local relapse or distant metastasis.

LAs possess analgesic and anti-inflammatory properties that indirectly improve cancer immunosurveillance. In addition, LAs have direct molecular effects on mitochondrial metabolism, generate oxidative stress, trigger apoptosis pathways in cancer cells and activate NK cells $(34,64)$. Preclinical studies found that treatment of cancer cells with clinically relevant concentrations of LAs inhibits their proliferation and migration or induces cell death (39). These direct antitumor effects described in many cancer cell lines are time- and concentration-dependent. In murine models, LAs showed a remarkable ability to decrease the incidence of metastases after surgery $(35,38)$. In humans, several clinical studies noticed that LAs used for extradural block attenuated the immunosuppressive endocrine effects generated by surgery (75). In addition, an array of retrospective trials and meta-analyses concluded that LAs used alone or in combination with general anesthesia preserved NK cell activity and improved overall survival and recurrence-free survival (18).

Several putative mechanisms may explain the antitumor properties of LAs. First, LAs reduce the immunosuppressive effects of surgery by reducing glucocorticoid stress and by dampening inflammation (88). Second, LAs stimulate the proliferation and the activity of NK cells that play an important role in the innate immune defense against cancer (83). Third, LAs have direct toxicity on cancer cells and may induce apoptosis before residual cancer cells migrate into adjacent tissues or reach the lumen of lymphatic or vascular capillaries. Finally, LAs reduce the consumption of major protumor molecules such as opioids and volatile agents during cancer surgery $(78,92)$. Preclinical data sustaining these findings are rather convincing as they have been reproduced in many cancer types. However, these promising data now need translation into the clinics. The outcome of ongoing 
randomized multicenter prospective trials dealing with the potential anticancer effects of LAs are urgently awaited. Indeed, the confirmation that LAs improve patient outcome would have a major impact on clinical practice, in particular in the context of oncological surgery.

\section{AUTHOR CONTRIBUTIONS}

AW provided the list of trials and designed the figures. OK helped for the design of figures. GK and LB wrote the manuscript. All authors contributed to the article and approved the submitted version.

\section{FUNDING}

OK is supported by Institut National du Cancer (INCa) and the DIM Elicit of the Ile-de-France; LB received a research grant by

\section{REFERENCES}

1. Ferlay J EM, Lam F, Colombet M, Mery L, Pineros M, et al. Global Cancer Observatory: Cancer Today (2020). Lyon: International Agency for Research on Cancer. Available at: https://gco.iarc.fr/today (Accessed February 2021). 2020.

2. Sullivan R, Alatise OI, Anderson BO, Audisio R, Autier P, Aggarwal A, et al. Global Cancer Surgery: Delivering Safe, Affordable, and Timely Cancer Surgery. Lancet Oncol (2015) 16(11):1193-224. doi: 10.1016/S1470-2045 (15)00223-5

3. Lambert AW, Pattabiraman DR, Weinberg RA. Emerging Biological Principles of Metastasis. Cell (2017) 168(4):670-91. doi: 10.1016/ j.cell.2016.11.037

4. Yang H, Xia L, Chen J, Zhang S, Martin V, Li Q, et al. Stress-GlucocorticoidTSC22D3 Axis Compromises Therapy-Induced Antitumor Immunity. Nat Med (2019) 25(9):1428-41. doi: 10.1038/s41591-019-0566-4

5. Tsuchiya Y, Sawada S, Yoshioka I, Ohashi Y, Matsuo M, Harimaya Y, et al. Increased Surgical Stress Promotes Tumor Metastasis. Surgery (2003) 133 (5):547-55. doi: 10.1067/msy.2003.141

6. Salmon P, Kaufman L. Preoperative Anxiety and Endocrine Response to Surgery. Lancet (1990) 335(8701):1340. doi: 10.1016/0140-6736(90) 91215-V

7. Ramirez MF, Ai D, Bauer M, Vauthey JN, Gottumukkala V, Kee S, et al. Innate Immune Function After Breast, Lung, and Colorectal Cancer Surgery. J Surg Res (2015) 194(1):185-93. doi: 10.1016/j.jss.2014.10.030

8. Vogelaar FJ, Abegg R, van der Linden JC, Cornelisse HG, van Dorsten FR, Lemmens VE, et al. Epidural Analgesia Associated With Better Survival in Colon Cancer. Int J Colorectal Dis (2015) 30(8):1103-7. doi: 10.1007/ s00384-015-2224-8

9. Holler JP, Ahlbrandt J, Burkhardt E, Gruss M, Rohrig R, Knapheide J, et al. Peridural Analgesia may Affect Long-Term Survival in Patients With Colorectal Cancer After Surgery (PACO-RAS-Study): An Analysis of a Cancer Registry. Ann Surg (2013) 258(6):989-93. doi: 10.1097/ SLA.0b013e3182915f61

10. Lin L, Liu C, Tan H, Ouyang H, Zhang Y, Zeng W. Anaesthetic Technique may Affect Prognosis for Ovarian Serous Adenocarcinoma: A Retrospective Analysis. Br J Anaesthesia (2011) 106(6):814-22. doi: 10.1093/bja/aer055

11. Zhang H, Yang L, Zhu X, Zhu M, Sun Z, Cata JP, et al. Association Between Intraoperative Intravenous Lidocaine Infusion and Survival in Patients Undergoing Pancreatectomy for Pancreatic Cancer: A Retrospective Study. Br J Anaesthesia (2020) 125(2):141-8. doi: 10.1016/ j.bja.2020.03.034
Bristol Myers Squibb Foundation France. AW was supported by El Programa Nacional de Becas "Don Carlos Antonio Lopez" (BECAL). GK is supported by the Ligue contre le Cancer (equipe labellisée); Agence National de la Recherche (ANR) - Projets blancs; AMMICa US23/CNRS UMS3655; Association pour la recherche sur le cancer (ARC); Association "Ruban Rose"; Cancéropôle Ile-de-France; Fondation pour la Recherche Médicale (FRM); a donation by Elior; Equipex Onco-PhenoScreen; European Joint Programme on Rare Diseases (EJPRD); Gustave Roussy Odyssea, the European Union Horizon 2020 Projects Oncobiome and Crimson; Fondation Carrefour; INCa; Inserm (HTE); Institut Universitaire de France; LabEx ImmunoOncology (ANR-18-IDEX-0001); the Leducq Foundation; a Cancer Research ASPIRE Award from the Mark Foundation; the RHU Torino Lumière; Seerave Foundation; SIRIC Stratified Oncology Cell DNA Repair and Tumor Immune Elimination (SOCRATE); and SIRIC Cancer Research and Personalized Medicine (CARPEM). This study contributes to the IdEx Universite de Paris ANR-18-IDEX-0001.
12. Wang X, Xie W, Gan S, Wang T, Chen X, Su D, et al. Effects of General Anesthesia Versus Local Anesthesia in Primary Hepatocellular Carcinoma Patients Presenting for Thermal Ablation Surgery: A Multiple Center Retrospective Cohort Study With Propensity Score Matching. Ann Trans Med (2020) 8(6):277. doi: 10.21037/atm.2020.03.88

13. Biki B, Mascha E, Moriarty DC, Fitzpatrick JM, Sessler DI, Buggy DJ. Anesthetic Technique for Radical Prostatectomy Surgery Affects Cancer Recurrence: A Retrospective Analysis. Anesthesiology (2008) 109(2):180-7. doi: 10.1097/ALN.0b013e31817f5b73

14. Exadaktylos AK, Buggy DJ, Moriarty DC, Mascha E, Sessler DI. Can Anesthetic Technique for Primary Breast Cancer Surgery Affect Recurrence or Metastasis? Anesthesiology (2006) 105(4):660-4. doi: 10.1097/00000542-200610000-00008

15. Chen WK, Miao CH. The Effect of Anesthetic Technique on Survival in Human Cancers: A Meta-Analysis of Retrospective and Prospective Studies. PloS One (2013) 8(2):e56540. doi: 10.1371/journal.pone.0056540

16. Pei L, Tan G, Wang L, Guo W, Xiao B, Gao X, et al. Comparison of Combined General-Epidural Anesthesia With General Anesthesia Effects on Survival and Cancer Recurrence: A Meta-Analysis of Retrospective and Prospective Studies. PloS One (2014) 9(12):e114667. doi: 10.1371/journal. pone.0114667

17. Sun Y, Li T, Gan TJ. The Effects of Perioperative Regional Anesthesia and Analgesia on Cancer Recurrence and Survival After Oncology Surgery: A Systematic Review and Meta-Analysis. Regional Anesthesia Pain Med (2015) 40(5):589-98. doi: 10.1097/AAP.0000000000000273

18. Weng M, Chen W, Hou W, Li L, Ding M, Miao C. The Effect of Neuraxial Anesthesia on Cancer Recurrence and Survival After Cancer Surgery: An Updated Meta-Analysis. Oncotarget (2016) 7(12):15262-73. doi: 10.18632/ oncotarget.7683

19. Strichartz GR. The Inhibition of Sodium Currents in Myelinated Nerve by Quaternary Derivatives of Lidocaine. J Gen Physiol (1973) 62(1):37-57. doi: 10.1085/jgp.62.1.37

20. D’Agostino G, Saporito A, Cecchinato V, Silvestri Y, Borgeat A, Anselmi L, et al. Lidocaine Inhibits Cytoskeletal Remodelling and Human Breast Cancer Cell Migration. Br J Anaesthesia (2018) 121(4):962-8. doi: 10.1016/ j.bja.2018.07.015

21. Jiang Y, Gou H, Zhu J, Tian S, Yu L. Lidocaine Inhibits the Invasion and Migration of TRPV6-Expressing Cancer Cells by TRPV6 Downregulation. Oncol Lett (2016) 12(2):1164-70. doi: 10.3892/ol.2016.4709

22. Mammoto T, Higashiyama S, Mukai M, Mammoto A, Ayaki M, Mashimo T, et al. Infiltration Anesthetic Lidocaine Inhibits Cancer Cell Invasion by Modulating Ectodomain Shedding of Heparin-Binding Epidermal Growth 
Factor-Like Growth Factor (HB-EGF). J Cell Physiol (2002) 192(3):351-8. doi: 10.1002/jcp.10145

23. Yin D, Liu L, Shi Z, Zhang L, Yang Y. Ropivacaine Inhibits Cell Proliferation, Migration and Invasion, Whereas Induces Oxidative Stress and Cell Apoptosis by Circscaf11/miR-145-5p Axis in Glioma. Cancer Manage Res (2020) 12:11145-55. doi: 10.2147/CMAR.S274975

24. Yoon JR, Whipple RA, Balzer EM, Cho EH, Matrone MA, Peckham M, et al. Local Anesthetics Inhibit Kinesin Motility and Microtentacle Protrusions in Human Epithelial and Breast Tumor Cells. Breast Cancer Res Treat (2011) 129(3):691-701. doi: 10.1007/s10549-010-1239-7

25. Dan J, Gong X, Li D, Zhu G, Wang L, Li F. Inhibition of Gastric Cancer by Local Anesthetic Bupivacaine Through Multiple Mechanisms Independent of Sodium Channel Blockade. Biomed Pharmacother Biomed Pharmacother (2018) 103:823-8. doi: 10.1016/j.biopha.2018.04.106

26. Zhang Y, Peng X, Zheng Q. Ropivacaine Inhibits the Migration of Esophageal Cancer Cells via Sodium-Channel-Independent But Prenylation-Dependent Inhibition of Rac1/JNK/Paxillin/FAK. Biochem Biophys Res Commun (2018) 501(4):1074-9. doi: 10.1016/j.bbrc.2018.05.110

27. Zheng Q, Peng X, Zhang Y. Cytotoxicity of Amide-Linked Local Anesthetics on Melanoma Cells via Inhibition of Ras and RhoA Signaling Independent of Sodium Channel Blockade. BMC Anesthesiol (2020) 20(1):43. doi: 10.1186/s12871-020-00957-4

28. Li C, Gao S, Li X, Li C, Ma L. Procaine Inhibits the Proliferation and Migration of Colon Cancer Cells Through Inactivation of the ERK/MAPK/ FAK Pathways by Regulation of RhoA. Oncol Res (2018) 26(2):209-17. doi: 10.3727/096504017X14944585873622

29. Zhang N, Xing X, Gu F, Zhou G, Liu X, Li B. Ropivacaine Inhibits the Growth, Migration and Invasion of Gastric Cancer Through Attenuation of WEE1 and PI3K/AKT Signaling via miR-520a-3p. OncoTargets Ther (2020) 13:5309-21. doi: 10.2147/OTT.S244550

30. Sui H, Lou A, Li Z, Yang J. Lidocaine Inhibits Growth, Migration and Invasion of Gastric Carcinoma Cells by Up-Regulation of miR-145. BMC Cancer (2019) 19(1):233. doi: 10.1186/s12885-019-5431-9

31. Sun H, Sun Y. Lidocaine Inhibits Proliferation and Metastasis of Lung Cancer Cell via Regulation of miR-539/EGFR Axis. Artif Cells Nanomed Biotechnol (2019) 47(1):2866-74. doi: 10.1080/21691401.2019.1636807

32. Ying B, Huang H, Li H, Song M, Wu S, Ying H. Procaine Inhibits Proliferation and Migration and Promotes Cell Apoptosis in Osteosarcoma Cells by Upregulation of MicroRNA-133b. Oncol Res (2017) 25(9):1463-70. doi: 10.3727/096504017X14878518291077

33. Piegeler T, Schlapfer M, Dull RO, Schwartz DE, Borgeat A, Minshall RD, et al. Clinically Relevant Concentrations of Lidocaine and Ropivacaine Inhibit TNFalpha-Induced Invasion of Lung Adenocarcinoma Cells In Vitro by Blocking the Activation of Akt and Focal Adhesion Kinase. Br J Anaesthesia (2015) 115(5):784-91. doi: 10.1093/bja/aev341

34. Qin A, Liu Q, Wang J. Ropivacaine Inhibits Proliferation, Invasion, Migration and Promotes Apoptosis of Papillary Thyroid Cancer Cells via Regulating ITGA2 Expression. Drug Dev Res (2020) 81(6):700-7. doi: $10.1002 /$ ddr.21671

35. Wada H, Seki S, Takahashi T, Kawarabayashi N, Higuchi H, Habu Y, et al. Combined Spinal and General Anesthesia Attenuates Liver Metastasis by Preserving TH1/TH2 Cytokine Balance. Anesthesiology (2007) 106(3):499-506. doi: 10.1097/00000542-200703000-00014

36. Johnson MZ, Crowley PD, Foley AG, Xue C, Connolly C, Gallagher HC, et al. Effect of Perioperative Lidocaine on Metastasis After Sevoflurane or Ketamine-Xylazine Anaesthesia for Breast Tumour Resection in a Murine Model. Br J Anaesthesia (2018) 121(1):76-85. doi: 10.1016/j.bja. 2017.12.043

37. Freeman J, Crowley PD, Foley AG, Gallagher HC, Iwasaki M, Ma D, et al. Effect of Perioperative Lidocaine, Propofol and Steroids on Pulmonary Metastasis in a Murine Model of Breast Cancer Surgery. Cancers (2019) 11(5):1-12. doi: 10.3390/cancers11050613

38. Wall TP, Crowley PD, Sherwin A, Foley AG, Buggy DJ. Effects of Lidocaine and Src Inhibition on Metastasis in a Murine Model of Breast Cancer Surgery. Cancers (2019) 11(10):1-10. doi: 10.3390/cancers11101414

39. Chen J, Jiao Z, Wang A, Zhong W. Lidocaine Inhibits Melanoma Cell Proliferation by Regulating ERK Phosphorylation. J Cell Biochem (2019) 120 (4):6402-8. doi: $10.1002 / j \mathrm{cb} .27927$
40. Tada M, Imazeki F, Fukai K, Sakamoto A, Arai M, Mikata R, et al. Procaine Inhibits the Proliferation and DNA Methylation in Human Hepatoma Cells. Hepatol Int (2007) 1(3):355-64. doi: 10.1007/s12072-007-9014-5

41. Sakaguchi M, Kuroda Y, Hirose M. The Antiproliferative Effect of Lidocaine on Human Tongue Cancer Cells With Inhibition of the Activity of Epidermal Growth Factor Receptor. Anesthesia Analgesia (2006) 102 (4):1103-7. doi: 10.1213/01.ane.0000198330.84341.35

42. Xing W, Chen DT, Pan JH, Chen YH, Yan Y, Li Q, et al. Lidocaine Induces Apoptosis and Suppresses Tumor Growth in Human Hepatocellular Carcinoma Cells In Vitro and in a Xenograft Model In Vivo. Anesthesiology (2017) 126(5):868-81. doi: 10.1097/ALN.0000000000001528

43. Ye L, Zhang Y, Chen YJ, Liu Q. Anti-Tumor Effects of Lidocaine on Human Gastric Cancer Cells In Vitro. Bratislavske Lekarske Listy (2019) 120(3):2127. doi: 10.4149/BLL_2019_036

44. Zhang L, Hu R, Cheng Y, Wu X, Xi S, Sun Y, et al. Lidocaine Inhibits the Proliferation of Lung Cancer by Regulating the Expression of GOLT1A. Cell Prolif (2017) 50(5):1-8. doi: 10.1111/cpr.12364

45. Le Gac G, Angenard G, Clement B, Laviolle B, Coulouarn C, Beloeil H. Local Anesthetics Inhibit the Growth of Human Hepatocellular Carcinoma Cells. Anesth Analg (2017) 125(5):1600-9. doi: 10.1213/ANE.0000000000002429

46. Castelli V, Piroli A, Marinangeli F, d'Angelo M, Benedetti E, Ippoliti R, et al. Local Anesthetics Counteract Cell Proliferation and Migration of Human Triple-Negative Breast Cancer and Melanoma Cells. J Cell Physiol (2020) 235 (4):3474-84. doi: 10.1002/jcp.29236

47. Kwakye AK, Kampo S, Lv J, Ramzan MN, Richard SA, Falagan AA, et al. Levobupivacaine Inhibits Proliferation and Promotes Apoptosis of Breast Cancer Cells by Suppressing the PI3K/Akt/mTOR Signalling Pathway. BMC Res Notes (2020) 13(1):386. doi: 10.1186/s13104-020-05191-2

48. Arita K, Utsumi T, Kato A, Kanno T, Kobuchi H, Inoue B, et al. Mechanism of Dibucaine-Induced Apoptosis in Promyelocytic Leukemia Cells (HL-60). Biochem Pharmacol (2000) 60(7):905-15. doi: 10.1016/S0006-2952(00) 00406-8

49. Jose C, Hebert-Chatelain E, Dias Amoedo N, Roche E, Obre E, Lacombe D, et al. Redox Mechanism of Levobupivacaine Cytostatic Effect on Human Prostate Cancer Cells. Redox Biol (2018) 18:33-42. doi: 10.1016/j.redox. 2018.05.014

50. Gong X, Dan J, Li F, Wang L. Suppression of Mitochondrial Respiration With Local Anesthetic Ropivacaine Targets Breast Cancer Cells. J Thorac Dis (2018) 10(5):2804-12. doi: 10.21037/jtd.2018.05.21

51. Okamoto A, Tanaka M, Sumi C, Oku K, Kusunoki M, Nishi K, et al. The Antioxidant N-Acetyl Cysteine Suppresses Lidocaine-Induced Intracellular Reactive Oxygen Species Production and Cell Death in Neuronal SH-SY5Y Cells. BMC Anesthesiol (2016) 16(1):104. doi: 10.1186/s12871-016-0273-3

52. Lirk P, Berger R, Hollmann MW, Fiegl H. Lidocaine Time- and DoseDependently Demethylates Deoxyribonucleic Acid in Breast Cancer Cell Lines In Vitro. Br J Anaesthesia (2012) 109(2):200-7. doi: 10.1093/bja/aes128

53. Villar-Garea A, Fraga MF, Espada J, Esteller M. Procaine is a DNADemethylating Agent With Growth-Inhibitory Effects in Human Cancer Cells. Cancer Res (2003) 63(16):4984-9.

54. Sabit H, Samy MB, Said OA, El-Zawahri MM. Procaine Induces Epigenetic Changes in HCT116 Colon Cancer Cells. Genet Res Int (2016) 2016:8348450. doi: $10.1155 / 2016 / 8348450$

55. Li YC, Wang Y, Li DD, Zhang Y, Zhao TC, Li CF. Procaine is a Specific DNA Methylation Inhibitor With Anti-Tumor Effect for Human Gastric Cancer. J Cell Biochem (2018) 119(2):2440-9. doi: 10.1002/jcb.26407

56. Gao Z, Xu Z, Hung MS, Lin YC, Wang T, Gong M, et al. Procaine and Procainamide Inhibit the Wnt Canonical Pathway by Promoter Demethylation of WIF-1 in Lung Cancer Cells. Oncol Rep (2009) 22 (6):1479-84. doi: 10.3892/or_00000590

57. Lirk P, Hollmann MW, Fleischer M, Weber NC, Fiegl H. Lidocaine and Ropivacaine, But Not Bupivacaine, Demethylate Deoxyribonucleic Acid in Breast Cancer Cells. Vitro Br J Anaesthesia (2014) 113(Suppl 1):i32-8. doi: 10.1093/bja/aeu201

58. Chen D, Yan Y, Xie J, Pan J, Chen Y, Li Q, et al. Amide-Type Local Anesthetics may Suppress Tumor Cell Proliferation and Sensitize Human Hepatocellular Carcinoma Cells to Cisplatin via Upregulation of RASSF1A Expression and Demethylation. J Cancer (2020) 11(24):7312-9. doi: 10.7150/ jca. 46630 
59. Xia W, Wang L, Yu D, Mu X, Zhou X. Lidocaine Inhibits the Progression of Retinoblastoma In Vitro and In Vivo by Modulating the Mir520a3p/EGFR Axis. Mol Med Rep (2019) 20(2):1333-42. doi: 10.3892/mmr.2019.10363

60. Li T, Chen L, Zhao H, Wu L, Masters J, Han C, et al. Both Bupivacaine and Levobupivacaine Inhibit Colon Cancer Cell Growth But Not Melanoma Cells In Vitro. J Anesthesia (2019) 33(1):17-25. doi: 10.1007/s00540-0182577-6

61. Tat T, Jurj A, Selicean C, Pasca S, Ionescu D. Antiproliferative Effects of Propofol and Lidocaine on the Colon Adenocarcinoma Microenvironment. J BUON Off J Balkan Union Oncol (2019) 24(1):106-15.

62. Chang YC, Liu CL, Chen MJ, Hsu YW, Chen SN, Lin CH, et al. Local Anesthetics Induce Apoptosis in Human Breast Tumor Cells. Anesthesia Analgesia (2014) 118(1):116-24. doi: 10.1213/ANE.0b013e3182a94479

63. Chang YC, Hsu YC, Liu CL, Huang SY, Hu MC, Cheng SP. Local Anesthetics Induce Apoptosis in Human Thyroid Cancer Cells Through the Mitogen-Activated Protein Kinase Pathway. PloS One (2014) 9(2): e89563. doi: 10.1371/journal.pone.0089563

64. Wang HW, Wang LY, Jiang L, Tian SM, Zhong TD, Fang XM. AmideLinked Local Anesthetics Induce Apoptosis in Human Non-Small Cell Lung Cancer. J Thorac Dis (2016) 8(10):2748-57. doi: 10.21037/jtd.2016.09.66

65. Lu J, Xu SY, Zhang QG, Xu R, Lei HY. Bupivacaine Induces Apoptosis via Mitochondria and P38 MAPK Dependent Pathways. Eur J Pharmacol (2011) 657(1-3):51-8. doi: 10.1016/j.ejphar.2011.01.055

66. Mirshahidi S, Shields TG, de Necochea-Campion R, Yuan X, Janjua A, Williams NL, et al. Bupivacaine and Lidocaine Induce Apoptosis in Osteosarcoma Tumor Cells. Clin Orthopaedics Related Res (2021) 479 (1):180-94. doi: 10.1097/CORR.0000000000001510

67. Wang W, Zhu M, Xu Z, Li W, Dong X, Chen Y, et al. Ropivacaine Promotes Apoptosis of Hepatocellular Carcinoma Cells Through Damaging Mitochondria and Activating Caspase-3 Activity. Biol Res (2019) 52(1):36. doi: 10.1186/s40659-019-0242-7

68. Zhang X, Pang W, Liu H, Wang J. Lidocine Potentiates the Cytotoxicity of 5Fluorouracil to Choriocarcinoma Cells by Downregulating ABC Transport Proteins Expression. J Cell Biochem (2019) 120(10):16533-42. doi: 10.1002/ jcb. 28913

69. Liang Y, Ji J, Lin Y, He Y, Liu J. The Ganglioside GM-1 Inhibits BupivacaineInduced Neurotoxicity in Mouse Neuroblastoma Neuro2a Cells. Cell Biochem Funct (2016) 34(6):455-62. doi: 10.1002/cbf.3208

70. Xuan W, Zhao H, Hankin J, Chen L, Yao S, Ma D. Local Anesthetic Bupivacaine Induced Ovarian and Prostate Cancer Apoptotic Cell Death and Underlying Mechanisms In Vitro. Sci Rep (2016) 6:26277. doi: 10.1038/ srep26277

71. Zhang H, Lin J, Hu T, Ren Z, Wang W, He Q. Effect of miR-132 on Bupivacaine-Induced Neurotoxicity in Human Neuroblastoma Cell Line. J Pharmacol Sci (2019) 139(3):186-92. doi: 10.1016/j.jphs.2019.01.014

72. Bernabe DG, Tamae AC, Biasoli ER, Oliveira SH. Stress Hormones Increase Cell Proliferation and Regulates Interleukin-6 Secretion in Human Oral Squamous Cell Carcinoma Cells. Brain Behav Immun (2011) 25(3):574-83. doi: 10.1016/j.bbi.2010.12.012

73. Yang EV, Kim SJ, Donovan EL, Chen M, Gross AC, Webster Marketon JI, et al. Norepinephrine Upregulates VEGF, IL-8, and IL-6 Expression in Human Melanoma Tumor Cell Lines: Implications for Stress-Related Enhancement of Tumor Progression. Brain Behav Immun (2009) 23 (2):267-75. doi: 10.1016/j.bbi.2008.10.005

74. Baker GH, Irani MS, Byrom NA, Nagvekar NM, Wood RJ, Hobbs JR, et al. Stress, Cortisol Concentrations, and Lymphocyte Subpopulations. Br Med J (1985) 290(6479):1393. doi: 10.1136/bmj.290.6479.1393

75. Carli F, Webster J, Pearson M, Pearson J, Bartlett S, Bannister P, et al. Protein Metabolism After Abdominal Surgery: Effect of 24-H Extradural Block With Local Anaesthetic. Br J Anaesthesia (1991) 67(6):729-34. doi: 10.1093/bja/ 67.6.729

76. Liu S, Carpenter RL, Neal JM. Epidural Anesthesia and Analgesia. Their Role in Postoperative Outcome. Anesthesiology (1995) 82(6):1474-506. doi: 10.1097/00000542-199506000-00019

77. Whelan P, Morris PJ. Immunological Responsiveness After Transurethral Resection of the Prostate: General Versus Spinal Anaesthetic. Clin Exp Immunol (1982) 48(3):611-8.
78. Kochhar A, Banday J, Ahmad Z, Panjiar P, Vajifdar H. Cervical Epidural Analgesia Combined With General Anesthesia for Head and Neck Cancer Surgery: A Randomized Study. J Anaesthesiol Clin Pharmacol (2020) 36 (2):182-6. doi: 10.4103/joacp.JOACP_72_19

79. Bar-Yosef S, Melamed R, Page GG, Shakhar G, Shakhar K, Ben-Eliyahu S. Attenuation of the Tumor-Promoting Effect of Surgery by Spinal Blockade in Rats. Anesthesiology (2001) 94(6):1066-73. doi: 10.1097/00000542200106000-00022

80. Sacerdote P, Manfredi B, Bianchi M, Panerai AE. Intermittent But Not Continuous Inescapable Footshock Stress Affects Immune Responses and Immunocyte Beta-Endorphin Concentrations in the Rat. Brain Behav Immun (1994) 8(3):251-60. doi: 10.1006/brbi.1994.1023

81. Ben-Eliyahu S, Page GG, Yirmiya R, Shakhar G. Evidence That Stress and Surgical Interventions Promote Tumor Development by Suppressing Natural Killer Cell Activity. Int J Cancer (1999) 80(6):880-8. doi: 10.1002/ (SICI)1097-0215(19990315)80:6<880::AID-IJC14>3.0.CO;2-Y

82. Lin E, Calvano SE, Lowry SF. Inflammatory Cytokines and Cell Response in Surgery. Surgery (2000) 127(2):117-26. doi: 10.1067/msy.2000.101584

83. Ramirez MF, Tran P, Cata JP. The Effect of Clinically Therapeutic Plasma Concentrations of Lidocaine on Natural Killer Cell Cytotoxicity. Regional Anesthesia Pain Med (2015) 40(1):43-8. doi: 10.1097/AAP.0000000000000191

84. Jaura AI, Flood G, Gallagher HC, Buggy DJ. Differential Effects of Serum From Patients Administered Distinct Anaesthetic Techniques on Apoptosis in Breast Cancer Cells In Vitro: A Pilot Study. Br J Anaesthesia (2014) 113 Suppl 1:i63-7. doi: 10.1093/bja/aet581

85. Dong H, Zhang Y, Xi H. The Effects of Epidural Anaesthesia and Analgesia on Natural Killer Cell Cytotoxicity and Cytokine Response in Patients With Epithelial Ovarian Cancer Undergoing Radical Resection. J Int Med Res (2012) 40(5):1822-9. doi: 10.1177/030006051204000520

86. Wang HL, Yan HD, Liu YY, Sun BZ, Huang R, Wang XS, et al. Intraoperative Intravenous Lidocaine Exerts a Protective Effect on CellMediated Immunity in Patients Undergoing Radical Hysterectomy. Mol Med Rep (2015) 12(5):7039-44. doi: 10.3892/mmr.2015.4235

87. Zhou D, Gu FM, Gao Q, Li QL, Zhou J, Miao CH. Effects of Anesthetic Methods on Preserving Anti-Tumor T-Helper Polarization Following Hepatectomy. World J Gastroenterol (2012) 18(24):3089-98. doi: 10.3748/ wjg.v18.i24.3089

88. Deegan CA, Murray D, Doran P, Moriarty DC, Sessler DI, Mascha E, et al. Anesthetic Technique and the Cytokine and Matrix Metalloproteinase Response to Primary Breast Cancer Surgery. Regional Anesthesia Pain Med (2010) 35(6):490-5. doi: 10.1097/AAP.0b013e3181ef4d05

89. Sultan SS. Paravertebral Block can Attenuate Cytokine Response When it Replaces General Anesthesia for Cancer Breast Surgeries. Saudi J Anaesthesia (2013) 7(4):373-7. doi: 10.4103/1658-354X.121043

90. O'Riain SC, Buggy DJ, Kerin MJ, Watson RWG, Moriarty DC. Inhibition of the Stress Response to Breast Cancer Surgery by Regional Anesthesia and Analgesia Does Not Affect Vascular Endothelial Growth Factor and Prostaglandin E2. Anesthesia Analgesia (2005) 100(1):244-9. doi: 10.1213/ 01.ANE.0000143336.37946.7D

91. Looney M, Doran P, Buggy DJ. Effect of Anesthetic Technique on Serum Vascular Endothelial Growth Factor C and Transforming Growth Factor Beta in Women Undergoing Anesthesia and Surgery for Breast Cancer. Anesthesiology (2010) 113(5):1118-25. doi: 10.1097/ ALN.0b013e3181f79a69

92. Kuo CP, Jao SW, Chen KM, Wong CS, Yeh CC, Sheen MJ, et al. Comparison of the Effects of Thoracic Epidural Analgesia and I.V. Infusion With Lidocaine on Cytokine Response, Postoperative Pain and Bowel Function in Patients Undergoing Colonic Surgery. Br J Anaesthesia (2006) 97(5):6406. doi: 10.1093/bja/ael217

93. Hayden JM, Oras J, Block L, Thorn SE, Palmqvist C, Salehi S, et al. Intraperitoneal Ropivacaine Reduces Time Interval to Initiation of Chemotherapy After Surgery for Advanced Ovarian Cancer: Randomised Controlled Double-Blind Pilot Study. Br J Anaesthesia (2020) 124(5):562-70. doi: 10.1016/j.bja.2020.01.026

94. Zhao T, Xia WH, Zheng MQ, Lu CQ, Han X, Sun YJ. Surgical Excision Promotes Tumor Growth and Metastasis by Promoting Expression of MMP-9 and VEGF in a Breast Cancer Model. Exp Oncol (2008) 30(1):60-4. 
95. Maccio A, Madeddu C. Inflammation and Ovarian Cancer. Cytokine (2012) 58(2):133-47. doi: 10.1016/j.cyto.2012.01.015

96. Wang Y, Yang J, Gao Y, Du Y, Bao L, Niu W, et al. Regulatory Effect of E2, IL-6 and IL-8 on the Growth of Epithelial Ovarian Cancer Cells. Cell Mol Immunol (2005) 2(5):365-72.

97. Browning L, Patel MR, Horvath EB, Tawara K, Jorcyk CL. IL-6 and Ovarian Cancer: Inflammatory Cytokines in Promotion of Metastasis. Cancer Manage Res (2018) 10:6685-93. doi: 10.2147/CMAR.S179189

98. Li K, Yang J, Han X. Lidocaine Sensitizes the Cytotoxicity of Cisplatin in Breast Cancer Cells via Up-Regulation of RARbeta2 and RASSF1A Demethylation. Int J Mol Sci (2014) 15(12):23519-36. doi: 10.3390/ijms151223519

99. Zhu G, Zhang L, Dan J, Zhu Q. Differential Effects and Mechanisms of Local Anesthetics on Esophageal Carcinoma Cell Migration, Growth, Survival and Chemosensitivity. BMC Anesthesiol (2020) 20(1):126. doi: 10.1186/s12871020-01039-1

100. Freeman J, Crowley PD, Foley AG, Gallagher HC, Iwasaki M, Ma D, et al. Effect of Perioperative Lidocaine and Cisplatin on Metastasis in a Murine Model of Breast Cancer Surgery. Anticancer Res (2018) 38(10):5599-606. doi: 10.21873 /anticanres.12894

101. Viale M, Vannozzi MO, Mandys V, Esposito M. Time-Dependent Influence of Procaine Hydrochloride on Cisplatin Antitumor Activity in P388 Tumor Bearing Mice. Anticancer Res (2001) 21(1A):485-7.

102. Esposito M, Fulco RA, Collecchi P, Zicca A, Cadoni A, Merlo F, et al. Improved Therapeutic Index of Cisplatin by Procaine Hydrochloride. J Natl Cancer Inst (1990) 82(8):677-84. doi: 10.1093/jnci/82.8.677

103. Cummings KC 3rd, Xu F, Cummings LC, Cooper GS. A Comparison of Epidural Analgesia and Traditional Pain Management Effects on Survival and Cancer Recurrence After Colectomy: A Population-Based Study. Anesthesiology (2012) 116(4):797-806. doi: 10.1097/ALN.0b013e31824674f6

104. Zimmitti G, Soliz J, Aloia TA, Gottumukkala V, Cata JP, Tzeng CW, et al. Positive Impact of Epidural Analgesia on Oncologic Outcomes in Patients Undergoing Resection of Colorectal Liver Metastases. Ann Surg Oncol (2016) 23(3):1003-11. doi: 10.1245/s10434-015-4933-1

105. Hiller JG, Hacking MB, Link EK, Wessels KL, Riedel BJ. Perioperative Epidural Analgesia Reduces Cancer Recurrence After Gastro-Oesophageal Surgery. Acta Anaesthesiol Scandinavica (2014) 58(3):281-90. doi: 10.1111/aas.12255

106. Ismail H, Ho KM, Narayan K, Kondalsamy-Chennakesavan S. Effect of Neuraxial Anaesthesia on Tumour Progression in Cervical Cancer Patients Treated With Brachytherapy: A Retrospective Cohort Study. Br J Anaesthesia (2010) 105(2):145-9. doi: 10.1093/bja/aeq156

107. Wu HL, Tai YH, Mandell MS, Tsou MY, Yang SH, Chen TH, et al. Effect of Epidural Analgesia on Cancer Prognosis After Colon Cancer Resection: A Single-Centre Cohort Study in Taiwan. BMJ Open (2020) 10(10):e036577. doi: 10.1136/bmjopen-2019-036577

108. Wurster EF, Pianka F, Warschkow R, Antony P, Brenner T, Weigand MA, et al. Peridural Analgesia Does Not Impact Survival in Patients After Colon Cancer Resection: A Retrospective Propensity Score-Adjusted Analysis. Int J Colorectal Dis (2019) 34(7):1283-93. doi: 10.1007/s00384-019-03315-0

109. Gottschalk A, Ford JG, Regelin CC, You J, Mascha EJ, Sessler DI, et al. Association Between Epidural Analgesia and Cancer Recurrence After Colorectal Cancer Surgery. Anesthesiology (2010) 113(1):27-34. doi: 10.1097/ALN.0b013e3181de6d0d

110. Gupta A, Bjornsson A, Fredriksson M, Hallbook O, Eintrei C. Reduction in Mortality After Epidural Anaesthesia and Analgesia in Patients Undergoing Rectal But Not Colonic Cancer Surgery: A Retrospective Analysis of Data From 655 Patients in Central Sweden. Br J Anaesthesia (2011) 107(2):164-70. doi: 10.1093/bja/aer100

111. Day A, Smith R, Jourdan I, Fawcett W, Scott M, Rockall T. Retrospective Analysis of the Effect of Postoperative Analgesia on Survival in Patients After Laparoscopic Resection of Colorectal Cancer. Br J Anaesthesia (2012) 109 (2):185-90. doi: 10.1093/bja/aes106

112. Merquiol F, Montelimard AS, Nourissat A, Molliex S, Zufferey PJ. Cervical Epidural Anesthesia is Associated With Increased Cancer-Free Survival in Laryngeal and Hypopharyngeal Cancer Surgery: A Retrospective PropensityMatched Analysis. Regional Anesthesia Pain Med (2013) 38(5):398-402. doi: 10.1097/AAP.0b013e31829cc3fb

113. Gottschalk A, Brodner G, Van Aken HK, Ellger B, Althaus S, Schulze HJ. Can Regional Anaesthesia for Lymph-Node Dissection Improve the Prognosis in
Malignant Melanoma? Br J Anaesthesia (2012) 109(2):253-9. doi: 10.1093/ bja/aes176

114. Cata JP, Gottumukkala V, Thakar D, Keerty D, Gebhardt R, Liu DD. Effects of Postoperative Epidural Analgesia on Recurrence-Free and Overall Survival in Patients With Nonsmall Cell Lung Cancer. J Clin Anesthesia (2014) 26 (1):3-17. doi: 10.1016/j.jclinane.2013.06.007

115. Lacassie HJ, Cartagena J, Branes J, Assel M, Echevarria GC. The Relationship Between Neuraxial Anesthesia and Advanced Ovarian Cancer-Related Outcomes in the Chilean Population. Anesthesia Analgesia (2013) 117 (3):653-60. doi: 10.1213/ANE.0b013e3182a07046

116. Wuethrich PY, Hsu Schmitz SF, Kessler TM, Thalmann GN, Studer UE, Stueber F, et al. Potential Influence of the Anesthetic Technique Used During Open Radical Prostatectomy on Prostate Cancer-Related Outcome: A Retrospective Study. Anesthesiology (2010) 113(3):570-6. doi: 10.1097/ ALN.0b013e3181e4f6ec

117. Forget P, Tombal B, Scholtes JL, Nzimbala J, Meulders C, Legrand C, et al. Do Intraoperative Analgesics Influence Oncological Outcomes After Radical Prostatectomy for Prostate Cancer? Eur J Anaesthesiol (2011) 28(12):830-5. doi: 10.1097/EJA.0b013e32834b7d9a

118. Binczak M, Tournay E, Billard V, Rey A, Jayr C. Major Abdominal Surgery for Cancer: Does Epidural Analgesia Have a Long-Term Effect on Recurrence-Free and Overall Survival? Annales Francaises D'anesthesie Reanimation (2013) 32(5):e81-8. doi: 10.1016/j.annfar.2013.02.027

119. Guerrero Orriach JL, Raigon Ponferrada A, Malo Manso A, Herrera Imbroda B, Escalona Belmonte JJ, Ramirez Aliaga M, et al. Anesthesia in Combination With Propofol Increases Disease-Free Survival in Bladder Cancer Patients Who Undergo Radical Tumor Cystectomy as Compared to Inhalational Anesthetics and Opiate-Based Analgesia. Oncology (2020) 98(3):161-7. doi: $10.1159 / 000504807$

120. Christopherson R, James KE, Tableman M, Marshall P, Johnson FE. LongTerm Survival After Colon Cancer Surgery: A Variation Associated With Choice of Anesthesia. Anesth Analg (2008) 107(1):325-32. doi: 10.1213/ ane.0b013e3181770f55

121. Deegan CA, Murray D, Doran P, Ecimovic P, Moriarty DC, Buggy DJ. Effect of Anaesthetic Technique on Oestrogen Receptor-Negative Breast Cancer Cell Function In Vitro. Br J Anaesthesia (2009) 103(5):685-90. doi: 10.1093/ bja/aep261

122. Wu YF, Li XP, Yu YB, Chen L, Jiang CB, Li DY, et al. Postoperative Local Incision Analgesia for Acute Pain Treatment in Patients With Hepatocellular Carcinoma. Rev Da Associacao Med Brasileira (2018) 64(2):175-80. doi: 10.1590/1806-9282.64.02.175

123. Finn DM, Ilfeld BM, Unkart JT, Madison SJ, Suresh PJ, Sandhu NPS, et al. Post-Mastectomy Cancer Recurrence With and Without a Continuous Paravertebral Block in the Immediate Postoperative Period: A Prospective Multi-Year Follow-Up Pilot Study of a Randomized, Triple-Masked, Placebo-Controlled Investigation. J Anesthesia (2017) 31(3):374-9. doi: 10.1007/s00540-017-2345-z

124. Sessler DI, Pei L, Huang Y, Fleischmann E, Marhofer P, Kurz A, et al. Recurrence of Breast Cancer After Regional or General Anaesthesia: A Randomised Controlled Trial. Lancet (2019) 394(10211):1807-15. doi: 10.1016/S0140-6736(19)32313-X

125. Karmakar MK, Samy W, Lee A, Li JW, Chan WC, Chen PP, et al. Survival Analysis of Patients With Breast Cancer Undergoing a Modified Radical Mastectomy With or Without a Thoracic Paravertebral Block: A 5-Year Follow-Up of a Randomized Controlled Trial. Anticancer Res (2017) 37 (10):5813-20.

126. Kim SY, Kim NK, Baik SH, Min BS, Hur H, Lee J, et al. Effects of Postoperative Pain Management on Immune Function After Laparoscopic Resection of Colorectal Cancer: A Randomized Study. Medicine (2016) 95 (19):e3602. doi: 10.1097/MD.0000000000003602

127. Xu ZZ, Li HJ, Li MH, Huang SM, Li X, Liu QH, et al. Epidural AnesthesiaAnalgesia and Recurrence-Free Survival After Lung Cancer Surgery: A Randomized Trial. Anesthesiology (2021) 135(3):419-32. doi: 10.1097/ ALN.0000000000003873

128. Tsui BC, Rashiq S, Schopflocher D, Murtha A, Broemling S, Pillay J, et al. Epidural Anesthesia and Cancer Recurrence Rates After Radical Prostatectomy. Can J Anaesthesia J Canadien D'anesthesie (2010) 57 (2):107-12. doi: 10.1007/s12630-009-9214-7 
129. Myles PS, Peyton P, Silbert B, Hunt J, Rigg JR, Sessler DI, et al. Perioperative Epidural Analgesia for Major Abdominal Surgery for Cancer and Recurrence-Free Survival: Randomised Trial. Bmj (2011) 342:d1491. doi: 10.1136/bmj.d1491

130. Du YT, Li YW, Zhao BJ, Guo XY, Feng Y, Zuo MZ, et al. Long-Term Survival After Combined Epidural-General Anesthesia or General Anesthesia Alone: Follow-Up of a Randomized Trial. Anesthesiology (2021) 135(2):233-45. doi: 10.1097/ALN.0000000000003835

131. Svendsen MN, Werther K, Nielsen HJ, Kristjansen PE. VEGF and Tumour Angiogenesis. Impact of Surgery, Wound Healing, Inflammation and Blood Transfusion. Scand J Gastroenterol (2002) 37(4):373-9.

132. Liu H, Dilger JP, Lin J. Effects of Local Anesthetics on Cancer Cells. Pharmacol Ther (2020) 212:107558. doi: 10.1016/j.pharmthera.2020.107558

133. Muller SD, Ziegler JSH, Piegeler T. Local Anesthetics and Recurrence After Cancer Surgery-What's New? A Narrative Review. J Clin Med (2021) 10 (4):1-14. doi: $10.3390 / \mathrm{jcm} 10040719$

Conflict of Interest: OK is scientific co-founder of Samsara Therapeutics; GK has been holding research contracts with Daiichi Sankyo, Eleor, Kaleido, Lytix Pharma, PharmaMar, Samsara, Sanofi, Sotio, Vascage and Vasculox/Tioma. GK is on the Board of Directors of the Bristol Myers Squibb Foundation France. GK is a scientific co-founder of everImmune, Samsara Therapeutics and Therafast Bio.
GK is the inventor of patents covering therapeutic targeting of aging, cancer, cystic fibrosis and metabolic disorders.

The remaining authors declare that the research was conducted in the absence of any commercial or financial relationships that could be construed as a potential conflict of interest.

The funders were not involved in the study design, collection, analysis, interpretation of data, the writing of this article or the decision to submit it for publication.

Publisher's Note: All claims expressed in this article are solely those of the authors and do not necessarily represent those of their affiliated organizations, or those of the publisher, the editors and the reviewers. Any product that may be evaluated in this article, or claim that may be made by its manufacturer, is not guaranteed or endorsed by the publisher.

Copyright (C) 2022 Wu Chuang, Kepp, Kroemer and Bezu. This is an open-access article distributed under the terms of the Creative Commons Attribution License (CC BY). The use, distribution or reproduction in other forums is permitted, provided the original author(s) and the copyright owner(s) are credited and that the original publication in this journal is cited, in accordance with accepted academic practice. No use, distribution or reproduction is permitted which does not comply with these terms. 